\title{
REPORT
}

\section{Highlights from the 'Noncoding RNA world: From Mechanism to Therapy' conference, July 21-23, 2021}

\author{
MONA KAMAL SAADELDIN ${ }^{1,2}$ \\ ${ }^{1}$ Department of Biosciences, University of Milan, I-20133 Milan, Italy; ${ }^{2}$ School of Science \\ and Engineering, American University in Cairo, New Cairo, Cairo 11835, Egypt
}

Received September 9, 2021; Accepted October 13, 2021

DOI: $10.3892 /$ wasj.2021.135

\begin{abstract}
Non-coding RNAs (ncRNAs) are involved in different cellular processes and some of them are considered to play a role in the development of numerous diseases. The recent discovery of ncRNAs is the result of several experimental and bioinformatics studies. On July 21-23, 2021, the 'Noncoding RNA World: From Mechanism to Therapy' conference that was held virtually (originally planned to be held in Basel, Switzerland), gathered experts and researchers from countries worldwide to cover the latest achievements in ncRNA research, including the basic molecular mechanisms and structural biology, bioinformatics, biotechnology, disease development and therapeutics of ncRNAs. The meeting was dominated by a feeling of satisfaction and enthusiasm for the novel discoveries in the ncRNA field that is considered to be relatively new. Intriguingly, several discoveries are added daily beside numerous hurdles that are encountered or remain to be resolved in order for these findings to be translated into clinical practice.
\end{abstract}

\section{Introduction}

The full spectra of non-coding RNA (ncRNA) research, beginning from the basic molecular and structural biology of ncRNAs, and continuing to the involvement of ncRNAs in disease development and therapeutics, as well as the biotechnological applications of ncRNAs, were discussed by world-leading speakers at the 'Noncoding RNA World: From Mechanism to Therapy' conference that took place virtually

Correspondence to: Dr Mona Kamal Saadeldin, Department of Biosciences, University of Milan, Via Celoria 26, I-20133 Milan, Italy

E-mail: m.kamal@aucegypt.edu; mona.saadeldin@unimi.it

Key words: non-coding RNA, molecular mechanisms, diseases development, bioinformatics, therapeutics, biotechnological applications, SARS-CoV-2 on July 21-23, 2021. Two keynote speakers; Professor Dr Narry Kim (Center of RNA Research, Institute for Basic Science, School of Biological Sciences, Seoul National University, Seoul, Korea) and Dr Piero Carninci (Deputy Director, RIKEN Center for Integrative Medical Sciences, Yokohama, Japan) participated in this conference. The participants with the best selected abstracts delivered oral presentations and the remaining abstracts were presented in interactive poster sessions. The conference was sponsored by Beckman Coulter, Inc. and Vector Builder and was organized by the MDPI non-coding RNA journal.

\section{ncRNAs shaping the future of humankind}

A large number of discoveries on ncRNAs have been recently demonstrated in various fields such as oncology, cardiology, virology, and others (1-6). ncRNAs are divided into different classes based on their size, architecture and mechanisms of action. MicroRNAs (miRNAs/miRs) are short RNAs of 22-23 nucleotides in length that bind to their target mRNAs, thus regulating protein translation via post-transcriptional gene silencing. Long ncRNAs (lncRNAs) are ncRNAs of $>200$ nucleotides in length; the functions of the majority of these have yet to be determined.

On the one hand, a wide overview of the importance of miRNAs was presented by Professor Dr Narry Kim; Professor Dr Kim reviewed the structure of miRNAs and the various related biogenesis pathways, namely the canonical (>99\%) and non-canonical pathways (DROSHA-independent and DICER-independent), as well as their functional relevance. The knowledge of the identity of miRNAs being canonical or non-canonical, and also which are the reliable annotations versus the false entries, were investigated in her laboratory using various approaches. The first approach relies on knowing the common defining features of pri-miRNAs (motifs recognized by DROSHA, DGCR8 and SRSF3). However, only a limited number of pri-miRNAs have all of these motifs. Thus, a second additional approach has been implemented; the dependence on biogenesis factors (DROSHA, DICER and XPO5), where they identified that DROSHA was critical and selective for the canonical miRNA 
pathway (7). Furthermore, Professor Dr Kim presented the collaborative research performed in her laboratory with the group of Professor Jae-Sung Woo which involved studying the atomic interactions of the microprocessor complex proteins (DROSHA and DGCRs) through the crystallization of the human microprocessor structure, apart from providing insight into the involvement of auxiliary cofactors (SRSF3, ERH and SAFB2) (8). They revealed the similarity between the structural organization of both proteins in terms of their catalytic motifs and central domains, although the two proteins differ as regards their primary sequences. The third approach is based on sequencing; formaldehyde cross-linking, immunoprecipitation and sequencing (fCLIP-seq) on DROSHA, which captured intact-ends products that lead to the mapping of cleavage sites. Nevertheless, further coverage was needed for a better understanding of miRNAs (9). This challenge was overcome using the fourth approach that uses a high throughput in vitro processing assay. They defined a cut-off value to separate the DROSHA-dependent from the DROSHA-independent miRNAs and identified 560 miRNAs $(30 \%)$ that are cleaved to produce pre-miRNAs. The remaining miRNAs $(\sim 70 \%)$ undergo unproductive processing; inverse processing or 'nick' processing, which are frequent and widespread for at least some pri-miRNAs in vitro, but are inconsistent in vivo based on fCLIP-seq data. This point was explained by the role of a cellular broad-acting factor; the SRSF3 cofactor that blocks the unproductive processing through the CNNC motif in new hairpins. Thus, in summary, their study provided a quantitative and comprehensive map of human pri-miRNA processing sites, and together the data and methods they produced are considered useful for the miRNA field.

On the other hand, Dr Piero Carninci indicated that lncRNAs are key players in regulating gene expression, apart from being extensively expressed in the human genome. Thus, it is eminent to understand their mechanisms of action. Dr Carninci provided an overview of the Capped Analysis of Gene Expression (CAGE) technology his group developed, which recognizes transcription start sites (TSSs) overlapping several structures, with a specific interest in regulatory IncRNAs; in addition, it can quantify their activities in the genome. Moreover, he illustrated how this technology was implicated in the functional annotation of the mammalian genome 5 (FANTOM5) project on a variety of samples types to determine their function. He subsequently revealed their systematic approach in FANTOM6 in order to provide a deeper understanding of the functions of lncRNAs by launching a large dataset built on silencing 285 lncRNAs in human primary fibroblasts (10). As an application of this technology, he discussed the SINEUPs, a novel group of non-coding antisense lncRNAs that enhance the translation of proteins transcribed from the region they overlap and can be used as a targeted therapy to rescue the altered gene expression. He indicated that the future is for genomics at the single-cell level and in accordance, his group is currently building a regulatory and transcriptional map at the single-cell level in the Human Cell Atlas (HCA) project which will facilitate the comprehension of biology (11). Eventually, an overview of novel advancement in ncRNAs in different disciplines was provided by the speakers. Since currently, SARS-CoV-2 is a hot topic worldwide, scientists from different fields are interested in extending their research to unveiling its mystery by applying different approaches and techniques (12). Some of these interesting studies were highlighted at this conference.

\section{ncRNA molecular and structural biology advancements}

Dr Xiaohua Shen (Tsinghua University, Beijing, China) demonstrated that the Phase Separation of RNA-Binding Protein (RBP) enhances the RNA Polymerase II (Pol II) involvement in the process of transcription and boosts the process. Transcription occurs in a chain of events that entails the congregation of a pre-initiation complex (PIC) and Pol II. RBPs which constitute $>62 \%$ of the chromatin proteome were discovered to be considerably co-localized with Pol II in genomic hotspots inferring a possible mutual collaborative interaction, and thus contributing to the rate-limiting step of active transcription. He demonstrated that the silencing RBPs (PSPC1 and PTBP) using RNA inhibitors abrogated Pol II binding and subsequently, the transcription process in embryonic stem cells. PSPC1 interacts with RNA to enhance the CTD phosphorylation of the Pol II subunit; a vital regulatory mechanism for cell cycle progression via the action of CDK7 and CDK9. He indicated that among the new challenges will be the identification of the RBP composition at individual sites and determining the mechanisms through which this affects cell fate (13).

Dr Yue Wan (Associate Director, Genome Institute of Singapore, Singapore) provided an overview of the strategies that can be used to identify the RNA function of regulatory genes, beginning from the RNA structure, and providing an example on SARS-CoV-2. RNA molecules fold into diverse structures with various specific functions; however, it is still difficult to predict the RNA structures. Among the techniques used to study RNA structure, is PORE-cupine technology. This technique can differentiate among gene-linked isoforms that are associated with definitive gene regulation by measuring the current changes when the RNA molecules pass through the nanopore. Dr Wan discussed that in studying the architecture of SARS-CoV-2, it was revealed that the virus has numerous alternative structures inside the host cells although they share the same sequence. The SARS-CoV-2 end region interacts with various regions at the 5 ' end, suggesting the dominant isoform. She also referred to their interest in exploring SARS-CoV-2 RNA-host RNA interactions that demonstrated the ability of the virus, upon its entry into the cell, to seize part of the mRNA 2-o methylation, consequently reducing its availability to host cells and the viability of host cells.

\section{ncRNA bioinformatics advancements}

Inferring with the functions of RNA molecules from their predicted structures specificities has been a priority among scientists in the field. Professor Dr Janusz M. Bujnicki (Laboratory of Bioinformatics and Protein Engineering, International Institute of Molecular and Cell Biology, Warsaw, Poland) presented the approach of his research group to overcome the obstacles of the experimental determination of high-resolution ncRNA structure and interactions that 
render the majority of ncRNAs structurally unelucidated. Using the computational RNA 3D modeling approach, the identification of functioning regions of the RNA could be achieved $(14,15)$. However, one of the drawbacks of this approach is that it lacks accuracy. Therefore, he defined the caveats of how the structure of RNA is encoded in the sequence, how its shape changes and what regulates this shift, and finally, how the RNA recognizes and interacts with other molecules. The main goal of his research group was to integrate the available biochemical and biophysical data to the function of RNA molecules using SimRNA (16). His research group also applied this to SARS-CoV-2 by obtaining its secondary structure, defining the conserved elements in its RNA and predicting the pockets that could be targeted by drugs. Moreover, his research group predicted the possible RNA-protein interactions.

Dr Aiming Ren (Life Sciences Institute, Zhejiang University, Hangzhou, China) highlighted the use of the novel Pepper Fluorescent RNA and NAD+1 Riboswitches in revealing the RNA function from its structure. For the former, Dr Ren illustrated the mechanism of pepper activation and shed light onto its application. As regards the latter and based on the tertiary structure, Dr Ren founded the regulatory model of the NAD+1 riboswitch (17).

\section{ncRNAs in disease development and therapeutics advancements}

An interesting shift is the translation from the bench to clinical practice. ncRNAs are promising key players with dual roles that can function both as underlying causes of pathologies, as well as promising therapeutic options. In terms of a causative agent in various pathologies, an interesting speech was provided by Professor Dr Shinichi Nakagawa (RNA Biology Laboratory, Faculty of Pharmaceutical Sciences, Hokkaido University, Hokkaido, Japan) on how the nuclear ncRNA $4.5 \mathrm{SH}$, which is highly homologous to SINE B1 retrotransposon, regulates mouse development. $4.5 \mathrm{SH}$ regulates the localization of mRNAs with antisense SINE B1 (asB1s) by forming double-stranded RNAs (dsRNAs) with asB1s, and thus is preferentially retained in the nucleus and cannot be exported to the cytoplasm. It was concluded that $4.5 \mathrm{SH}$ inhibits asB1 mRNA and the $45 \mathrm{SH}$ cluster is essential for mouse development (18).

Another intriguing talk by Professor Dr Irene Bozzoni (Sapienza University of Rome, Rome, Italy) highlighted the role of ncRNAs in the pathophysiology of the amyotrophic lateral sclerosis (ALS) neurodegenerative disorder. Professor Dr Bozzoni discovered ncRNAs that regulate the shift from dividing progenitors into post-mitotic motor neurons (MNs) at the post-transcriptional level. Her research group identified several lncRNAs that are expressed in mature MNs that are free from ALS-associated mutations of the FUS protein. With the aid of in vitro and in vivo CRISPR/Cas9 mutants, they discovered regulatory circuitries by which ncRNAs affect MN differentiation and survival (19).

Arriving at the promising role of ncRNAs as a future therapeutic strategy, Professor Dr Thomas Thum [Institute of Molecular and Translational Therapeutic Strategies, Medizinische Hochschule Hannover (MHH), Hannover,
Germany] discussed the translational validation of the protective effect arising from inhibiting microRNA-132-3p (miR-132) that causes fibrosis and cardiac hypertrophy in a large animal model of myocardial infarction using CDR132L, an antisense oligonucleotide. The results of the research performed by his group revealed a dose-dependent improvement in heart functions in terms of increasing the ejection fraction and decreasing the amino terminal fragment of pro-brain natriuretic peptide (NT-proBNP). Furthermore, their study proved that miR-132 inhibition was a promising approach for the treatment of heart failure (HF) of non-ischemic origin (2). He then mentioned the leading clinical trial by his research group worldwide on patients with HF using CDR132L (NCT04045405); 28 patients were divided randomly to receive either CDR132L at increasing doses of $0.32,1,3$ and $10 \mathrm{mg} / \mathrm{kg}$ body weight or the placebo ( $0.9 \%$ saline) in two intravenous infusions with a gap of 4 weeks. CDR132L was demonstrated to be safe and to improve cardiac functions with a suggested effective dose level of $\geq 1 \mathrm{mg} / \mathrm{kg}$. The HF protective effect was dose-dependent and linear plasma levels with no signs of accumulation were attained (3).

Another interesting talk was provided by Professor Dr Sven Diederichs [Division of Cancer Research, Department of Thoracic Surgery, University of Freiburg, Freiburg, Germany; and Division of RNA Biology and Cancer, German Cancer Research Center (DKFZ), Heidelberg, Germany] where he reviewed the role of linc NMR IncRNAs in controlling nucleotide metabolism through the YBX1-RRM2 axis in cancer (20). They used the RNA dependence concept using the RNA-dependent protein assay (R-DeeP) that uses density gradient ultracentrifugation to characterize these RNA-dependent proteins (21). Using the R-DeeP screening, his research group discovered the CTCF transcription factor as entirely RNA-dependent and that RNA was required for CTCF-chromatin (22).

\section{ncRNA biotechnological advancements}

Professor Dr Wensheng Wei (Beijing Advanced Innovation Center for Genomics, Peking University, Beijing, China) highlighted their high throughput genomic deletion screening strategy for functional lncRNAs that leads to the discovery of novel lncRNA oncogenes or tumor suppressor genes (23). Furthermore, he introduced their new LEAPER gene-editing strategy that efficiently edits endogenous transcripts and can be used in the treatment of genetic diseases, providing an example of restoring $\alpha$-L iduronidase activity in fibroblasts obtained from patients with Hurler syndrome (24).

Finally, a speech was delivered by Dr Igor Ulitsky (Weizmann Institute of Science, Rehovot, Israel), discussing the peculiar structures in the 'NOncoding RNA Activated by DNA Damage (NORAD)' IncRNA that enables them to inhibit Pumilio activity (25). A pair of repeats from the central NORAD structure is responsible for inhibiting Pumilio, where the 5' module enhances the inhibition, while the $3^{\prime}$ limits it. The efficiency of reactivating Pumilio is associated with the number of PUMILIO response elements (PREs), where NORAD structure clusters PREs through inter and intramolecular interactions. Finally, FAM120A prevents the PREs degradation in G/C-rich contents. 


\section{Conclusion}

ncRNAs present a promising new strategy that can be implemented to provide solutions for numerous long-standing, as well as novel diseases that shape the human future. Several studies have led to revealing new horizons and paving the way for a promising trend of medicine that is capable of understanding the machinery through which the genome is regulated.

\section{Acknowledgements}

The author would like to express special thanks to Professor Dr Rory Johnson (Laboratory for Genomics of Long noncoding RNA and Disease, Department of Medical Oncology, Inselspital, Bern University Hospital, University of Bern, Switzerland and Department for BioMedical Research, University of Bern, Bern, Switzerland) and Professor Dr Ling-Ling Chen (State Key Laboratory of Molecular Biology, Shanghai Key Laboratory of Molecular Andrology, CAS Center for Excellence in Molecular Cell Science, Shanghai Institute of Biochemistry and Cell Biology, University of Chinese Academy of Sciences, Chinese Academy of Sciences, Shanghai, China; School of Life Science and Technology, Shanghai Tech University, Shanghai, China) for organizing the 'Noncoding RNA World: From Mechanism to Therapy' conference and their feedback support on this report.

\section{Funding}

No funding was received.

\section{Availability of data and materials}

Data sharing is not applicable to this article, as no datasets were generated or analyzed during the current study.

\section{Author's contribution}

The author MKS conceived, wrote, revised and edited the manuscript. The author MKS confirms the authenticity of all the raw data and has read and approved the final manuscript.

\section{Ethics approval and consent to participate}

Not applicable.

\section{Patient consent for publication}

Not applicable.

\section{Competing interests}

The author declares that there are no competing interests.

\section{References}

1. Yang Y, Ge H, Li DQ and Xu AX: E2F1-Induced lncRNA BAIAP2-AS1 overexpression contributes to the malignant progression of hepatocellular carcinoma via miR-361-3p/SOX4 axis. Dis Markers 2021: 6256369, 2021.
2. Hinkel R, Batkai S, Bähr A, Bozoglu T, Straub S, Borchert T, Viereck J, Howe A, Hornaschewitz N, Oberberger L, et al: AntimiR-132 attenuates myocardial hypertrophy in an animal model of percutaneous aortic constriction. J Am Coll Cardiol 77: 2923-2935, 2021.

3. Täubel J, Hauke W, Rump S, Viereck J, Batkai S, Poetzsch J, Rode L, Weigt H, Genschel C, Lorch U, et al: Novel antisense therapy targeting microRNA-132 in patients with heart failure: Results of a first-in-human Phase $1 \mathrm{~b}$ randomized, double-blind, placebo-controlled study. Eur Heart J 42: 178-188, 2021.

4. Martin SE, Gan H, Toomer G, Sridhar N and Sztuba-Solinska J: The m6A landscape of polyadenylated nuclear (PAN) RNA and its related methylome in the context of KSHV replication. RNA 27: 1102-1125, 2021.

5. Pereira M, Ribeiro DR, Pinheiro MM, Ferreira M, Kellner S and Soares AR: $\mathrm{m}^{5}$ U54 tRNA Hypomodification by Lack of TRMT2A Drives the Generation of tRNA-Derived Small RNAs. Int J Mol Sci 22: 2941, 2021.

6. Youness RA and Gad MZ: Long non-coding RNAs: Functional regulatory players in breast cancer. Noncoding RNA Res 4: 36-44, 2019. Erratum in: Noncoding RNA Res 5: 219, 2020.

7. Kim YK, Kim B and Kim VN: Re-evaluation of the roles of DROSHA, Export in 5, and DICER in microRNA biogenesis. Proc Natl Acad Sci USA 113: E1881-E1889, 2016.

8. Kwon SC, Nguyen TA, Choi YG, Jo MH, Hohng S, Kim VN and Woo JS: Structure of Human DROSHA. Cell 164: 81-90, 2016.

9. Kim B, Jeong K and Kim VN: Genome-wide Mapping of DROSHA Cleavage Sites on Primary MicroRNAs and Noncanonical Substrates. Mol Cell 66: 258-269.e5, 2017.

10. Ramilowski JA, Yip CW, Agrawal S, Chang JC, Ciani Y, Kulakovskiy IV, Mendez M, Ooi JLC, Ouyang JF, Parkinson N, et al. Functional annotation of human long noncoding RNAs via molecular phenotyping. Genome Res 30: 1060-1072, 2020.

11. Moody J, Kouno T, Suzuki A, Shibayama Y, Terao C, Chang JC, López-Redondo F, Yip CW, Ando Y, Yamamoto K, et al: Profiling of transcribed cis-regulatory elements in single cells. bioRxiv: doi: https://doi.org/10.1101/2021.04.04.438388.

12. Saadeldin MK, Abdel-Aziz AK and Abdellatif A: Dendritic cell vaccine immunotherapy; the beginning of the end of cancer and COVID-19. A hypothesis. Med Hypotheses 146: $110365,2021$.

13. Shao W, Bi X, Gao B, Wu J, Pan Y, Yin Y, Liu Z, Zhang W, Jiang X, Ren W, et al: Phase separation of RNA-binding protein promotes polymerase engagement and transcription. bioRxiv: doi: https://doi.org/10.1101/2021.03.26.436939.

14. Nithin C, Ghosh P and Bujnicki JM: Bioinformatics tools and benchmarks for computational docking and 3D structure prediction of RNA-protein complexes. Genes (Basel) 9: 432, 2018.

15. Ponce-Salvatierra A, Astha, Merdas K, Nithin C, Ghosh P, Mukherjee S and Bujnicki JM: Computational modeling of RNA 3D structure based on experimental data. Biosci Rep 39: BSR20180430, 2019

16. Boniecki MJ, Lach G, Dawson WK, Tomala K, Lukasz P, Soltysinski T, Rother KM and Bujnicki JM: SimRNA: A coarse-grained method for RNA folding simulations and 3D structure prediction. Nucleic Acids Res 44: e63, 2016.

17. Chen H, Egger M, Xu X, Flemmich L, Krasheninina O, Sun A, Micura R and Ren A: Structural distinctions between NAD+ riboswitch domains 1 and 2 determine differential folding and ligand binding. Nucleic Acids Res 48: 12394-12406, 2020.

18. Ishida K, Miyauchi K, Kimura Y, Mito M, Okada S, Suzuki T and Nakagawa S: Regulation of gene expression via retrotransposon insertions and the noncoding RNA 4.5S RNAH. Genes Cells 20: 887-901, 2015.

19. Errichelli L, Dini Modigliani S, Laneve P, Colantoni A, Legnini I, Capauto D, Rosa A, De Santis R, Scarfò R, Peruzzi G, et al: FUS affects circular RNA expression in murine embryonic stem cell-derived motor neurons. Nat Commun 8: 14741, 2017.

20. Gandhi M, Groß M, Holler JM, Coggins SAA, Patil N, Leupold JH, Munschauer M, Schenone M, Hartigan CR, Allgayer $\mathrm{H}$, et al: The lncRNA lincNMR regulates nucleotide metabolism via a YBX1-RRM2 axis in cancer. Nat Commun 11: $3214,2020$.

21. Caudron-Herger M, Rusin SF, Adamo ME, Seiler J, Schmid VK, Barreau E, Kettenbach AN and Diederichs S: R-DeeP: Proteome-wideandQuantitative Identification of RNA-Dependent Proteins by Density Gradient Ultracentrifugation. Mol Cell 75: 184-199.e10, 2019. 
22. Caudron-Herger M, Wassmer E, Nasa I, Schultz A-S, Seiler J, Kettenbach AN and Diederichs S: Identification, quantification and bioinformatic analysis of RNA-dependent proteins by RNase treatment and density gradient ultracentrifugation using R-DeeP. Nat Protoc 15: 1338-1370, 2020.

23. Zhu S, Li W, Liu J, Chen CH, Liao Q, Xu P, Xu H, Xiao T, Cao Z, Peng J, et al: Genome-scale deletion screening of human long non-coding RNAs using a paired-guide RNA CRISPR-Cas9 library. Nat Biotechnol 34: 1279-1286, 2016.
24. Qu L, Yi Z, Zhu S, Wang C, Cao Z, Zhou Z, Yuan P, Yu Y, Tian F, Liu Z, et al: Programmable RNA editing by recruiting endogenous ADAR using engineered RNAs. Nat Biotechnol 37: 1059-1069, 2019. Erratum in: Nat Biotechnol 37: 1380, 2019.

25. Tichon A, Perry RBT, Stojic L and Ulitsky I: SAM68 is required for regulation of Pumilio by the NORAD long noncoding RNA. Genes Dev 32: 70-78, 2018.

(i) (2) This work is licensed under a Creative Commons Attribution-NonCommercial-NoDerivatives 4.0 International (CC BY-NC-ND 4.0) License. 